\title{
Niveles de incremento y rendimiento maderable de poblaciones naturales de Pinus arizonica Engl., de EI Poleo, Chihuahua.
}

\author{
E. Marcelo Zepeda Bautista ${ }^{1}$ \\ Alfonso Domínguez Pereda ${ }^{2}$
}

\begin{abstract}
RESUMEN
Se presentan los resultados de un primer análisis dasométrico de datos de parcelas permanentes de observación silvícola, establecidas en la mesa de El Poleo, municipio de Madera, Chihuahua, a principios de la década de los 70's, consistentes en ecuaciones dasométricas que permiten conocer el comportamiento promedio, a través del tiempo, del incremento y rendimiento maderables y la sobrevivencia probable, de las masas arboladas representadas en los datos. Tales ecuaciones permiten conocer inicialmente los incrementos y rendimientos maderables probables netos de los rodales representados en la muestra, con la consiguiente ventaja para el manejo silvícola operativo de las masas arboladas de El Poleo, Madera, Chihuahua.
\end{abstract}

PALABRAS CLAVE:

Modelo compatible, crecimiento, ecuación dasométrica, Pinus arizonica. rendimiento, madera

\begin{abstract}
A first dasometric analysis using data from permanent plots was made around the early 70's at El Poleo, in Chihuahua State. The results consist of a set of dasometric equations allowing the estimation of the average behavior along time, of growth, yield and possible survival of the stands represented in the sample. Such equations initially allow us to know the net growth and yield wood of those stands, with the obvious advantage for operative silvicultural management of stands from El Poleo, Madera, Chihuahua.
\end{abstract}

KEY WORDS:

Compatible model, growth, yield, wood, dasometric equation, Pinus arizonica.

1 Universidad Autónoma de Chapingo. División de Ciencias Forestales. Apdo. Postal. 37. Chapingo, Edo. de México. C.P. 56230. México.

2 Unidad de Conservación y Desarrollo Forestal No. 2. El Largo-Madera, Chihuahua, México.

Manuscrito recibido para su publicación el 29 de Agosto de 1997 


\section{INTRODUCCION}

La apertura comercial inducida por la globalización de la economía mundial, obliga a México a identificar e instrumentar estrategias productivas que le permitan participar en la satisfacción de la demanda mundial y regional de productos forestales, en particular de los maderables.

Tal obligación se puede satisfacer, básicamente, a través del manejo intensivo de poblaciones arboladas, que posibilite al administrador del bosque, el control de algunos de los factores que gobiernan las respuestas del incremento y rendimiento maderable de dichas masas.

Debido principalmente a su ubicación latitudinal y clima, los bosques chihuahuenses no se distinguen por los altos rendimientos en sus especies maderables. Sin embargo, existen microcondiciones en las que los rendimientos son notables y para las que existe, afortunadamente, información dasometrica que puede ser usada en la construcción de modelos dasometricos de pronóstico, en una primera aproximación, que permitan preparar, más adelante, calendarios de cosecha basados en criterios de rentabilidad financiera.

Aún cuando la zona de El Poleo, Madera, Chih., ha sido sujeta desde hace más de 70 años a aprovechamientos maderables organizados, y se contaba con la información de incremento y rendimiento maderable necesaria para preparar los modelos dasométricos objeto de este trabajo, ello no se había hecho.

La investigación del incremento y rendimiento de la especie de interés, en su condición natural, proporcionará información que permita su comparación con los de otras especies y regiones forestales importantes, y la explicación de las relaciones entre rendimiento $y$ densidad, para la calidad de sitio en que crecen los rodales de interés, en la mesa de El Poleo, Chihuahua.

\section{OBJETIVOS}

El propóito de este estudio puede resumirse en los siguientes objetivos específicos:

a. Traducir algunos atributos dasométricos de la condición silvícola presente en los datos, en coeficientes de regresión muestral de ecuaciones con un cierto significado biológico, que permita entender las propiedades biológicas de la función de producción lograda.

b. Generar un sistema de ecuaciones para el estudio futuro de los efectos de la densidad neta de los rodales y la calidad de sitio, de la mesa de El Poleo, Chih., sobre el incremento y el rendimiento maderable de Pinus arizonica Engl.

c. Representar el comportamiento de la sobrevivencia de Pinus arizonica Engl., en El Poleo, a través del tiempo.

\section{METODOLOGIA \\ Características del área de estudio, (Salmón, 1980)}

Localización. La Mesa del Poleo, Municipio de Madera, se sitúa en terrenos de la Unidad de Conservación y Desarrollo (UCoDeFo) No. 2, la cual se localiza al Noroeste del Estado de Chihuahua, entre los meridianos $108^{\circ} 1^{\prime}$ y $108^{\circ} 45^{\prime}$, al Oeste de Greenwich y entre los paralelos $28^{\circ} 45^{\prime}$ y $30^{\circ} 00^{\prime}$ de latitud Norte. Políticamente se ubica en los municipios de Madera y Casas Grandes (Fig. 1).

Orografía e hidrografía. La UCoDeFo está situada en la Sierra Madre Occidental y sus terrenos son de aptitud forestal. Están constituidos por barrancas y 


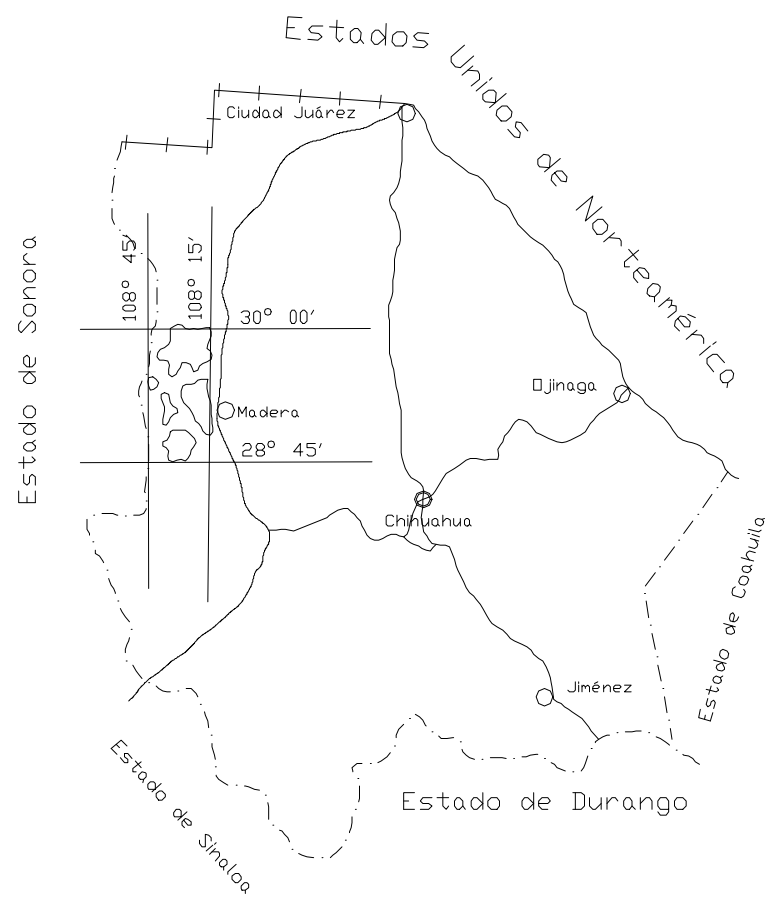

Figura 1. Ubicación del área de estudio en el Estado de Chihuahua (Salmón, 1980)

mesetas principalmente y sus altitudes varían entre los 1,200 y los 3,000 msnm.

Los terrenos son recorridos por arroyos y ríos que los cruzan en varias direcciones, con vertiente al Pacífico. Los ríos más importantes son el Papigochi, el Tutuaca y el Chico, afluentes del Aros. Los ríos Papigochi y Chico dividen la UCoDeFo en dos zonas geográficas o series: "El Largo" y "Madera". El río Colorado y el río Negro forman el río Gavilán y después el río Bavispe, el que se une al Aros y ambos forman el río Yaqui. Estos ríos son permanentes y muestran gastos sumamente reducidos durante la época de estiaje; sobre todo en la parte alta de la cuenca.

Suelos. Los suelos son de origen volcánico, de color café, rojo, gris y combinaciones de ellos. Su profundidad varía entre 0 y $165 \mathrm{~cm}$, con media en 45 $\mathrm{cm}$. Los materiales predominantes son rocas y lajas. Su textura es arcillosa, arcillo - arenosa, arcillo - limosa y limo arenosa.

Clima. La temperatura media máxima es de $10.4^{\circ} \mathrm{C}$ y la mínima de $2.0^{\circ} \mathrm{C}$. Los meses mas fríos son enero y febrero. Las heladas ocurren desde los primeros días del mes de octubre y hasta marzo, y se cuenta con 152 días, en promedio, libres de heladas. Los vientos corren en dirección SW, S y W. Su velocidad promedio es de 15.7 nudos por hora, con un mínimo de 10.0 y un máximo de 20.0.

El régimen de lluvias presenta una precipitación media anual de $813 \mathrm{~mm}$, con una mínima extrema de 402 y una máxima extrema de $1441 \mathrm{~mm}$. El promedio de días con lluvia en la zona es de 89.1, con precipitación media de 9.1 $\mathrm{mm}$ por día. El rango de variación extrema de días con lluvia va de 70 a 139. La precipitación se presenta en verano (abril a septiembre) y en invierno (octubre a marzo). 


\section{Características de las masas arboladas de El Poleo y variación de la muestra.} La mesa del Poleo está poblada por rodales puros, coetáneos, de origen natural, de Pinus arizonica Engl. y Pinus durangensis Mtz., predominando la primera especie. Su estructura vertical se compone de dos estratos: Un piso alto, relicto de la vegetación original, y un piso bajo constituido por poblaciones de segúndo crecimiento, creciendo en alta densidad. El origen del arbolado residual del dosel superior, producto del tipo de aprovechamiento al que otrora fueron sujetas las masas, imposibilita garantizar que el arbolado dominante actual lo haya sido siempre. La base de datos utilizada proviene de 11 parcelas experimentales (10, más un testigo) de $625 \mathrm{~m}^{2}(25 \times 25$ $\mathrm{m})$, con una franja de amortiguamiento de $10 \mathrm{~m}$ de ancho alrededor de cada parcela. Las parcelas, establecidas entre abril y octubre de 1970, están distribuídas en la mesa de El Poleo, un rodal excepcional de 360 hectáreas de extensión aunque el área de influencia de las parcelas, es de aproximadamente 1000 hectáreas de características similares a las de la mesa de interés, ubicadas a su alrededor.

\section{Lógica de construcción del modelo de incremento y rendimiento maderable.}

Para la construcción de las ecuaciones se empleó la metodología de Clutter (1963), omitiendo de las expresiones iniciales al indicador de calidad de sitio, ya que el modelo de incremento en área basal presentó inconsistencia en esa variable, pues el coeficiente de regresión muestral asociado al índice de sitio, además de no poseer significancia estadística aceptable, no presentó un signo adecuado. No habiendo manera de mantener a la variable "índice de sitio" en el modelo referido, para las condiciones actuales de la muestra, se procedió a eliminarla, sin que por ello la expresión resultante perdiera compatibilidad analítica y demás propiedades del enfoque metodológico adoptado; además de que la variable "índice de sitio" se incorporó finalmente en el modelo para estimar el volumen total (modelo 3). Las parcelas han sido sujetas al tratamiento del resto del bosque cada diez años, bajo el método de desarrollo silvícola, con un turno de 70 años y han sido remedidas cada cinco años, desde 1970 a la fecha.

En la tabla 1 se muestra el rango de variación de la muestra disponible para la obtención de las ecuaciones que sirven de base a este trabajo.

Tabla 1. Rango de variación de algunas variables dasométricas de las parcelas permanente de la mesa de Ael Poleo@Chihuahua.

\begin{tabular}{|l|c|c|c|c|c||}
\hline VARIABLE & $\mathrm{N}$ & MINIMO & MAXIMO & MEDIA & DESV. EST. \\
\hline Eprom & 50 & 25.0 & 45.0 & 35.4 & 7.2 \\
Hdom & 50 & 10.4 & 22.0 & 17.0 & 3.9 \\
IS & 50 & 19.0 & 30.9 & 25.6 & 2.3 \\
N1 & 39 & 448.0 & 5888.0 & 1392.0 & 1260.1 \\
N2 & 39 & 320.0 & 4992.0 & 1127.4 & 1024.7 \\
AB/ha & 72 & 5.7 & 45.7 & 25.0 & 8.8 \\
VOL/ha & 76 & 23.5 & 406.6 & 178.8 & 90.8 \\
\hline
\end{tabular}

N: Número de árboles por hectárea

Eprom: Edad promedio inicial (años)

Hdom: Altura dominante $(\mathrm{m})$

IS: Indice de sitio (m)

N1,N2: Número de árboles por hectárea en los tiempos uno y dos;

$\mathrm{Ab} / \mathrm{ha}$ : Area basal $\left(\mathrm{m}^{2} / \mathrm{ha}\right)$

VOL/ha: Existencias reales ( $\mathrm{m}^{3} / \mathrm{ha}$ ). 
Partiendo de que:

$$
d A B / d E=-A B[\ln (A B)] / E+C_{1}(A B / E)
$$

aplicando a (1) el procedimiento de Clutter (1963), se tiene que:

$$
\ln \left(A B_{2}\right)=\left(E_{1} / E_{2}\right)^{*} \ln \left(A B_{1}\right)+c_{1}\left(1-E_{1} / E_{2}\right)
$$

Una vez estimado el coeficiente $c_{1}$ de (2), (3) se ajustó al conjunto de datos disponible:

$$
\ln (V)=B_{0}+B_{1} I S+B_{2} / E+B_{3} \ln (A B)
$$

Los valores de IS se estimaron usando la expresión válida para Pinus arizonica Engl., de El Largo-Madera, Chih. (Zepeda et al., 1990):

$$
\text { IS }=133.7178\left[\text { Hdom/133.7178] }{ }^{(E / 60) 0.3207}\right.
$$

Derivando (3) con respecto al tiempo, se tiene que:

$$
d V / d E=V\left\{-B_{2} / E^{2}+B_{3}(d A B / d E) / A B\right\}
$$

De las expresiones anteriores, se tiene finalmente que:

$$
\ln \left(V_{2}\right)=b_{0}+b_{1} I S+b_{2} / E_{2}+b_{3}\left(E_{1} / E_{2}\right)^{*} \ln \left(A B_{1}\right)+b_{4}\left(1-E_{1} / E_{2}\right)
$$

Con (1), (2), (4) y (5) es posible estimar, además del incremento instantáneo en área basal y volumen, el área basal y el volumen en el futuro, a partir del área basal y edad actuales.

Nótese que sólo se ajustaron a los datos (2) y (3), y que (1), (4) y (5) se obtuvieron algebraicamente, lo que origina ciertos problemas estadísticos. Sin embargo, la anterior fue la única manera de ajustar las expresiones de interés al

$$
(\mathrm{dNo} / \mathrm{dE}) / \mathrm{No}=\mathrm{B}_{0}+\left(\mathrm{B}_{1} / \mathrm{E}\right)+\mathrm{B}_{2} \mathrm{IS}
$$

Expresión que resuelta corresponde a la siguiente ecuación de diferencia algebraica (Clutter et al.1983):

$$
N_{2}=\left\{N_{1}\left(E_{2} / E_{1}\right)^{B 1}\right\}\left\{\operatorname{Exp}\left[\left(B_{0}+B_{2} I S\right)\left(E_{2}-E_{1}\right)\right]\right\}
$$

Donde:

$\mathrm{dAB} / \mathrm{dE}=$ Tasa instantánea, corriente o anual, de incremento en área basal $\left(\mathrm{m}^{2} / \mathrm{ha} / \mathrm{año}\right)$ $\mathrm{AB}=$ Area basal $\left(\mathrm{m}^{2} / \mathrm{ha}\right)$; 


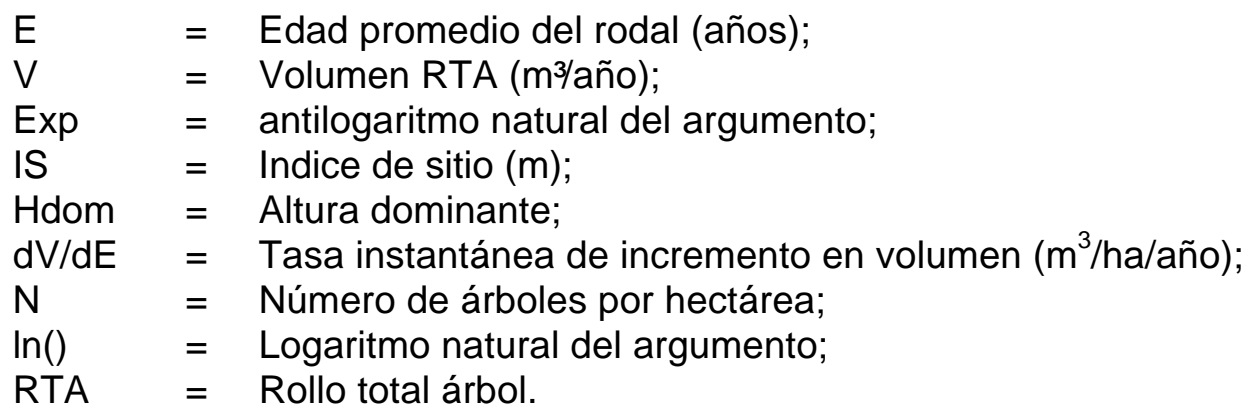

Los subíndices en $A B, E$ y $V$ y $N$, de las expresiones (2), (5) y (7), indican el inicio y final del período de estimación del atributo de interés.

\section{RESULTADOS Y DISCUSION}

A continuación se presentan las ecuaciones usadas en este trabajo. Para detalles sobre las mismas, se sugiere consultar el trabajo de Zepeda et al. (1994a).

$$
\begin{aligned}
& \ln (\mathrm{V})=2.029278+0.028849(\mathrm{IS})-29.089894 / \mathrm{E}+1.015761^{*} \ln (\mathrm{AB}) \\
& \ln \left(\mathrm{AB}_{2}\right)=\left(\mathrm{E}_{1} / \mathrm{E}_{2}\right)^{\star} \ln \left(\mathrm{AB}_{1}\right)+4.45\left(1-\mathrm{E}_{1} / \mathrm{E}_{2}\right) \\
& \ln \left(\mathrm{V}_{2}\right)=2.029288+0.028849(\mathrm{IS})-29.089894 / \mathrm{E}_{2}+1.015761\left(\mathrm{E}_{1} / \mathrm{E}_{2}\right)^{\star} \ln \left(\mathrm{AB}_{1}\right)+ \\
& 4.520136\left(1-\mathrm{E}_{1} / \mathrm{E}_{2}\right) \\
& \mathrm{dAB} / \mathrm{dE}=(\mathrm{AB}) / \mathrm{E}[4.45-\ln (\mathrm{AB})] \\
& \mathrm{dV} / \mathrm{dE}=\mathrm{V}\left\{+29.089894 / \mathrm{E}^{2}+1.0157611(\mathrm{dAB} / \mathrm{dE}) / \mathrm{AB}\right\} \\
& \mathrm{N}_{2}=\left\{\mathrm{N}_{1}\left(\mathrm{E}_{2} / \mathrm{E}_{1}\right)^{0.984457}\right\}\left\{\mathrm{Exp}\left[\left(0.0-0.003367^{\star} \mathrm{IS}\right)\left(\mathrm{E}_{2}-\mathrm{E}_{1}\right)\right]\right\}
\end{aligned}
$$

Donde los términos ya se definieron párrafos arriba

Las ecuaciones (8) y (9) se ajustaron a los datos, omitiendo valores de volumen inferiores a $55 \mathrm{~m}^{3} / \mathrm{ha}$. Por las limitaciones enunciadas de la muestra disponible, de momento las ecuaciones tienen sus mejores comportamientos sólo en los siguientes rangos: $A B: 5$ a 80 m²/ha; EDAD: 15 a 100 años; IS: 14 a 29 m; en particular la ecuación (13), ya que sobreestima considerablemente en edades inferiores a los 15 años.
El coeficiente de (9) se limitó(1) a 4.45 , ya que valores de éste iguales a 5 unidades, hacen suponer áreas basales de $148.41 \mathrm{~m}^{2} / \mathrm{ha}$, imposibles de lograr en la realidad si se considera, por ejemplo, que las plantaciones Esteafricanas de alto rendimiento exhiben áreas basales

1. La restricción a 4.45 no significa limitación alguna en la estructura o arquitectura de modelo; únicamente es un valor asintótico al que se restringe el cálculo. Si se desea se pueden emplear valores más grandes en dicho coeficiente según el juicio del usuario 
máximas de 64, 68 y $86 \mathrm{~m}^{2} /$ ha., para Cupressus sp., $P$. patula y $P$. radiata, respectivamente (Alder, 1979). En este caso se asumen áreas basales límite extremas de $85.62 \mathrm{~m}^{2} / \mathrm{ha}^{(2)}$, que se consideraron razonables.

El coeficiente de (8), para la variable IS, sugiere poca contribución de esa variable a la explicación de la variación total del volumen por hectárea, en la muestra usada en la construcción de las ecuaciones.

El coeficiente de (8), asociado al área basal, evidencia la relación alométrica existente entre ésta y el volumen por unidad de área, existente en masas homogéneas, y representa el valor de la elasticidad del volumen con respecto al área basal, por hectárea. La elasticidad de $Y$ con respecto a $X$, es el cambio en la tasa relativa de crecimiento de $\mathrm{Y}$, ante un cambio pequeño en la tasa relativa de crecimiento de $X$.

Los valores de los coeficientes de la ecuación de sobrevivencia muestran que la proporción de mortalidad es moderada en las poblaciones de $P$. arizonica, seguramente por sus rendimientos y velocidades de saturación de los espacios de crecimiento disponibles, también moderados.

En las figuras 2, 3 y 4 se muestra el comportamiento de algunas variables dasométricas, generado con las ecuaciones anteriores. Su comparación con los resultados de Zepeda et al. (1994a y b), evidencia los efectos de suponer $c_{1}$ en (2), mayor de 4.45. De tal comparación se desprende la bondad de suponer que $c_{1}=4.45$. Por su parte, en las figuras 5, 6, 7 y 8 , se presentan los comportamientos espaciales de algunas variables dasométricas de interés.

2 Zepeda et al. (1994a y b) usaron $4.5\left(90 \mathrm{~m}^{2} / \mathrm{ha}\right)$ satisfactoriamente)
El comportamiento de las diferentes ecuaciones es satisfactorio aunque las estimaciones están sujetas a las limitaciones de la muestra. Tales estimaciones no pueden considerarse pésimas, pero tampoco inmejorables. Los resultados de pronósticos, para basar decisiones silvícolas, deben usarse con las reservas del caso.

Como ejemplo de lo anterior, nótese que el incremento instantáneo en área basal, para el área basal límite seleccionada $\left(85.62 \mathrm{~m}^{2} / \mathrm{ha}\right)$, culmina en un nivel de densidad de alrededor de los $30 \mathrm{~m}^{2} /$ ha, aunque en edad no lo hace, pues la ecuación (9) no presenta un cambio notorio en su concavidad, conforme la edad tiende a cero (Fig 2). Esto deberá mejorarse más adelante.

El uso práctico de las ecuaciones aquí usadas debe limitarse, por ahora, a condiciones silvícolas en el rango de variación de las variables dasométricas presentadas en la tabla 1, en tanto no existan datos adicionales para calibrarlas. Las extrapolaciones deben tomarse con las reservas del caso.

Los resultados indicados en las figuras 2 y 3 , francamente extraordinarios para las condiciones del bosque Chihuahuense, e.g., existencias de 600 $\mathrm{m}^{3} /$ ha e incrementos instantáneos de aproximadamente $20 \mathrm{~m} / \mathrm{h}$ /año (con máximo a rededor de 20 años) y promedio

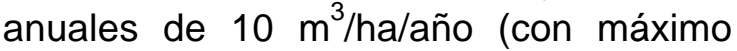
alrededor de los 30 años), deben tomarse con prudencia y tener presente que sólo corresponden a estimaciones procuradas por un conjunto de ecuaciones obtenidas a partir de la geometría particular de ciertos datos usados en su construcción, correspondientes a una muestra parcial de las condiciones ambientales $y$ silvícolas del lugar. 


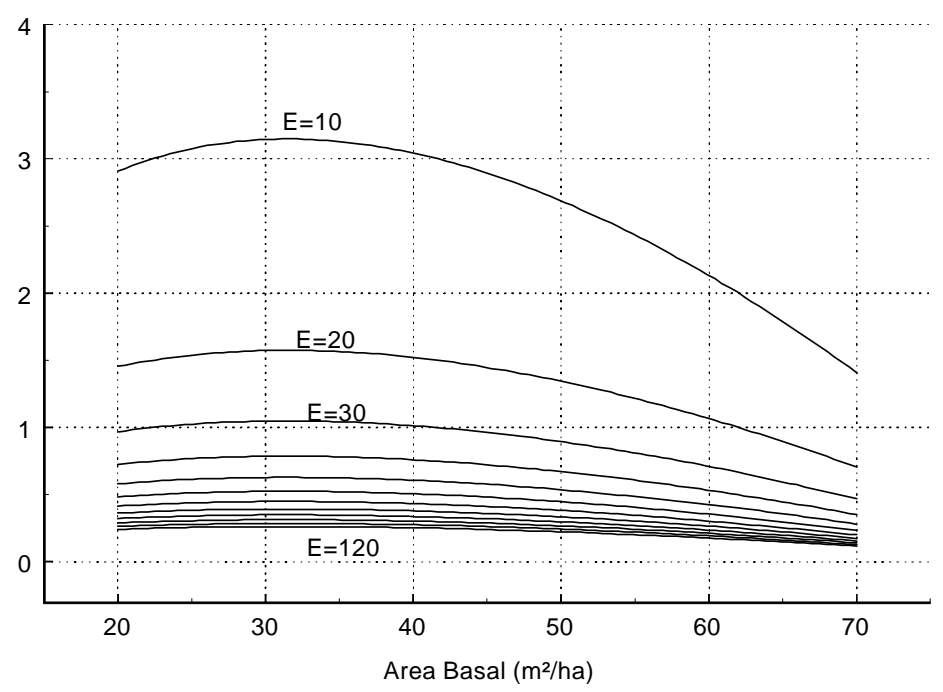

a) $\quad y=$ Incremento instantáneo en área basal ( $\left.\mathrm{m}^{2} / \mathrm{ha} / \mathrm{año}\right) ; \mathrm{x}=$ Area basal $\left(\mathrm{m}^{2} / \mathrm{ha}\right) ; \mathrm{z}=$ Edad (años); IS $=23 \mathrm{~m} ; \mathrm{Edad}=10-120$, cada 10 años; Niveles de área basal $=20-70 \mathrm{~m} / \mathrm{ha}$, con 4.45 en (2)

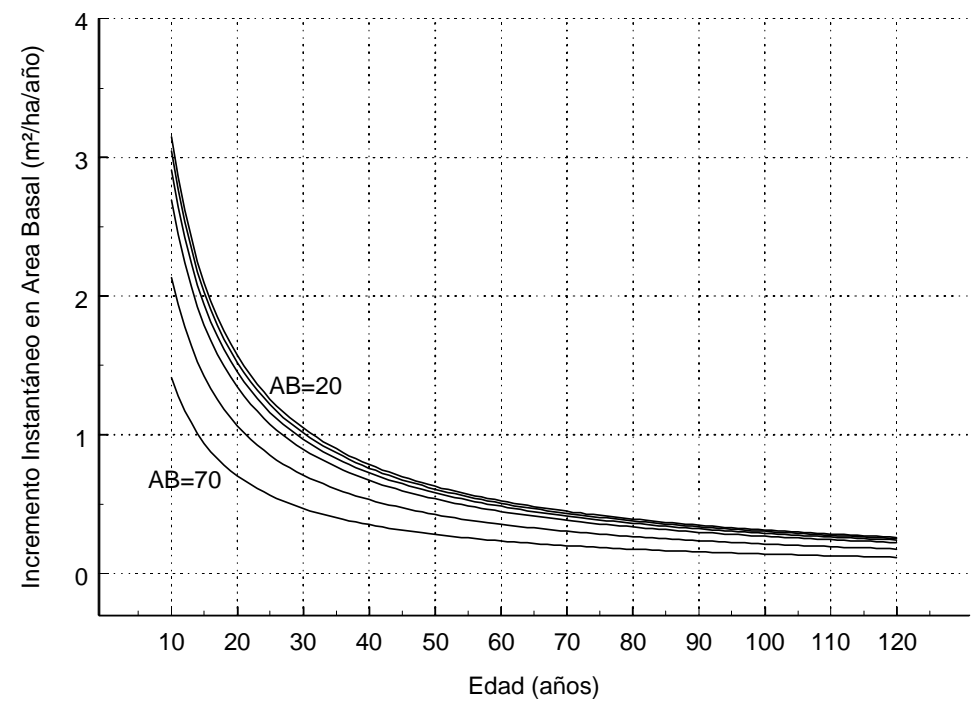

b) $\quad y=$ Inc. instantáneo en área basal ( $\left.\mathrm{m}^{2} / \mathrm{ha} / \mathrm{año}\right) ; \mathrm{x}=$ Edad (años); $\mathrm{z}=$ Nivel de área basal (años); IS $=23$ $\mathrm{m}$; Edad $=10-120$ años; Niveles de área basal $=20-70 \mathrm{~m}^{2} / \mathrm{ha}$, con 4.45 en (2)

Figura 2. Comportamiento del incremento instantáneo en área basal (a y b) de $P$. arizonica, en El Poleo, Chih. 


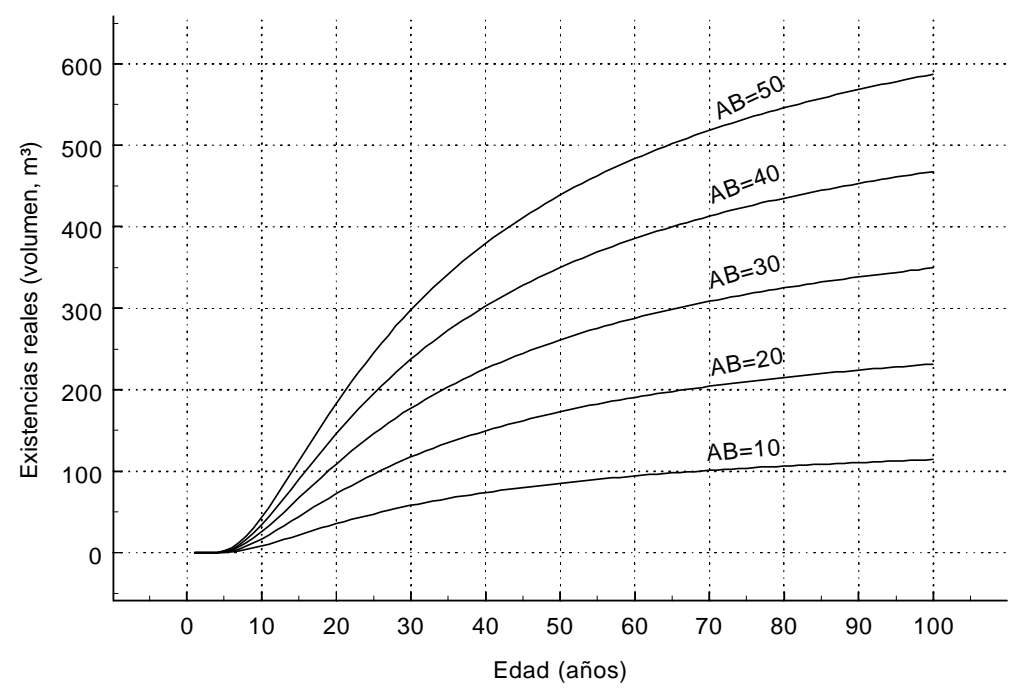

a) $y=$ Existencias reales ( $\mathrm{m}^{3} / \mathrm{ha}$ ); $\mathrm{x}=$ Edad (años); $\mathrm{z}=$ Nivel de área basal (años); IS $=23 \mathrm{~m}$; Edad $=10-100$ años; Niveles de área basal $=10-50 \mathrm{~m}^{2} / \mathrm{ha}$, con 4.45 en (2)

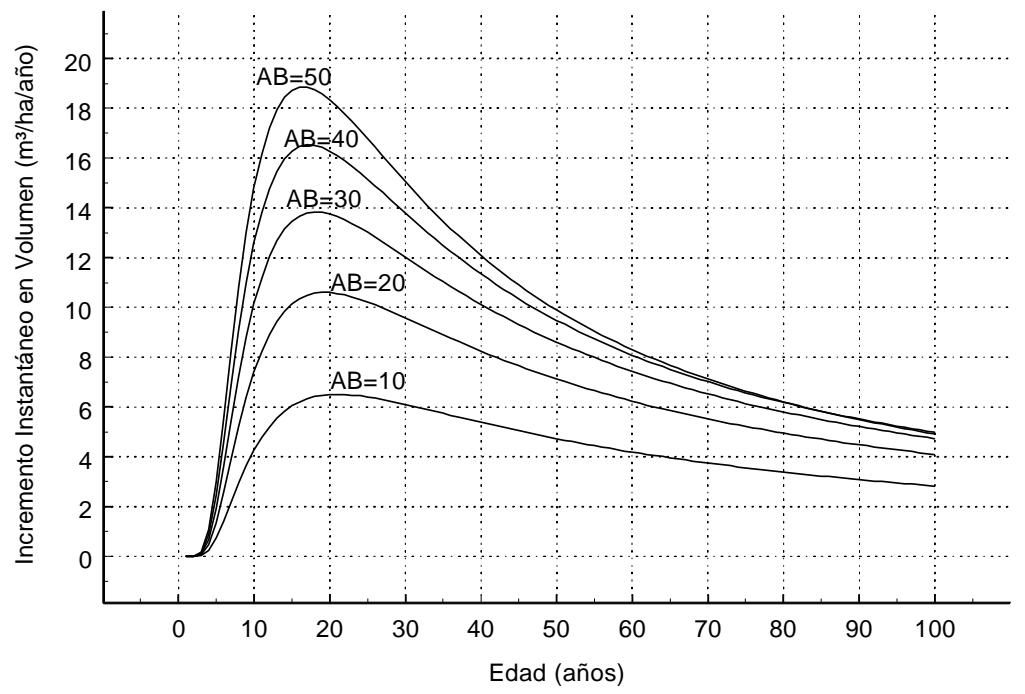

b) $y=$ Inc. instantáneo en volumen ( $\left.\mathrm{m}^{3} / \mathrm{ha} / \mathrm{año}\right) ; \mathrm{x}=$ Edad (años); $\mathrm{z}=$ Nivel de área basal (años); IS = $23 \mathrm{~m}$; Edad =1 -100 años; Niveles de área basal= $10-50 \mathrm{~m}^{2} / \mathrm{ha}$, con 4.45 en (2) 


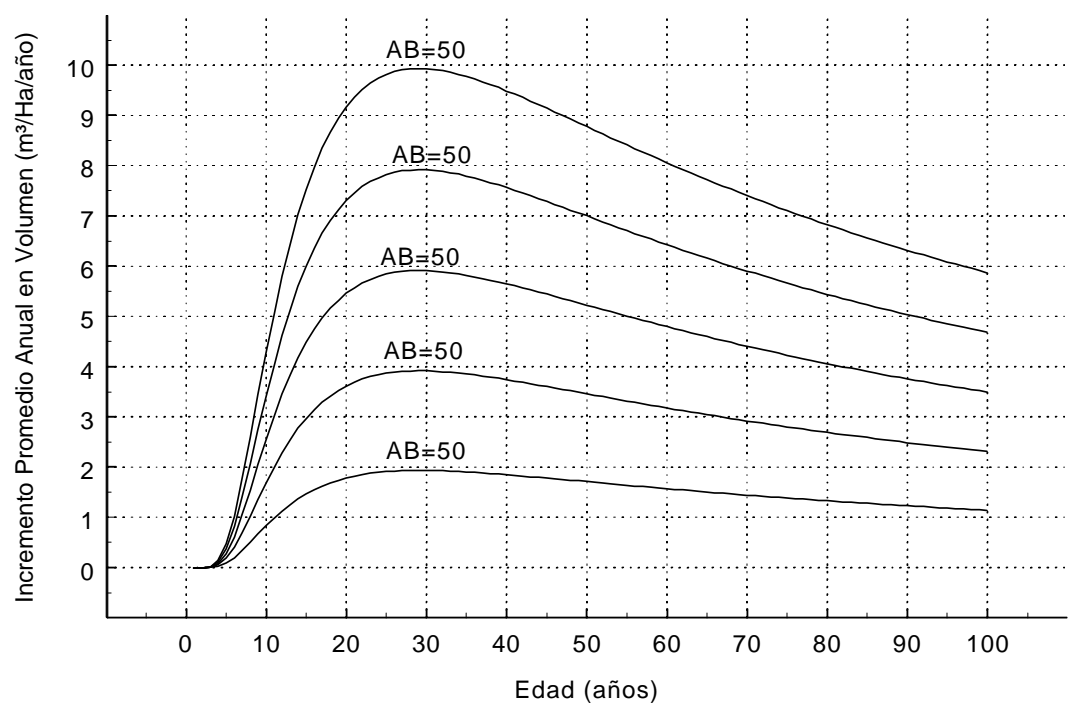

c) $\quad y=$ Inc. promedio anual en volumen ( $\left.\mathrm{m}^{3} / \mathrm{ha} / \mathrm{año}\right) ; \mathrm{x}=$ Edad (años); $\mathrm{z}=$ Nivel de área basal (años); IS $=23$ $\mathrm{m}$; Edad = 1 - 100; Niveles de área basal $=10-50 \mathrm{~m}^{2} / \mathrm{ha}$, con 4.45 en (2)

Figura 3. Comportamiento de volumen (a), incremento instantáneo en volumen (b) e incremento promedio anual en volumen (c), de $P$. arizonica, en El Poleo, Chih.

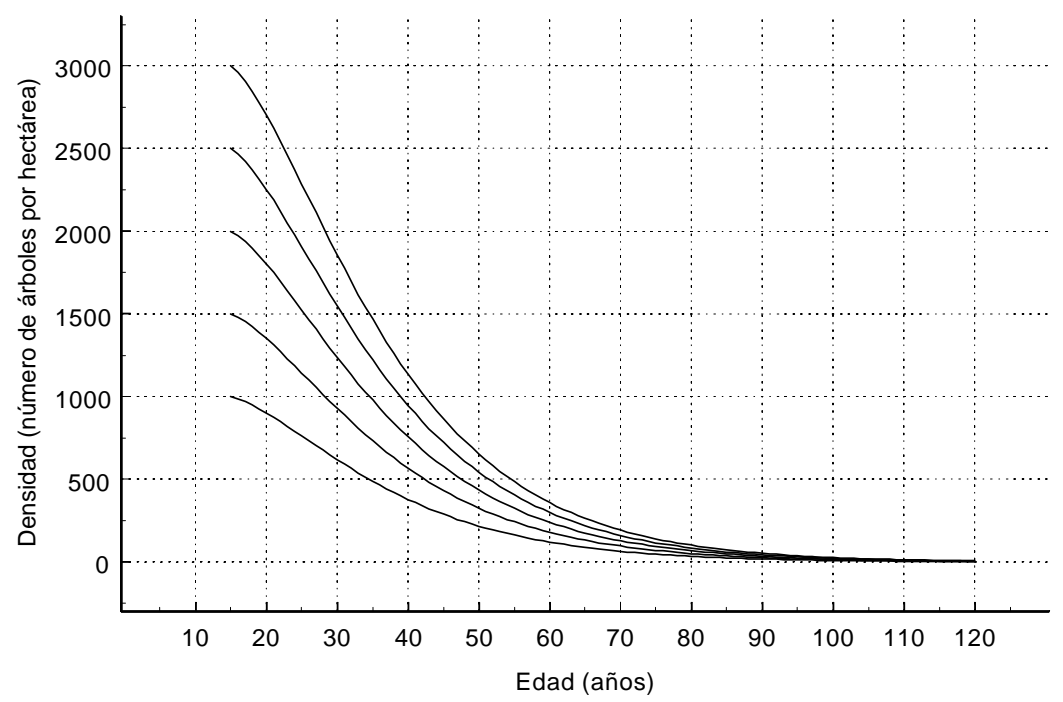

IS $=23 \mathrm{~m} ; \mathrm{Edad}=20-12$ años

Figura 4. Comportamiento de la sobrevivencia (árboles por hectárea) de $P$. arizonica, en El Poleo, Chih. 


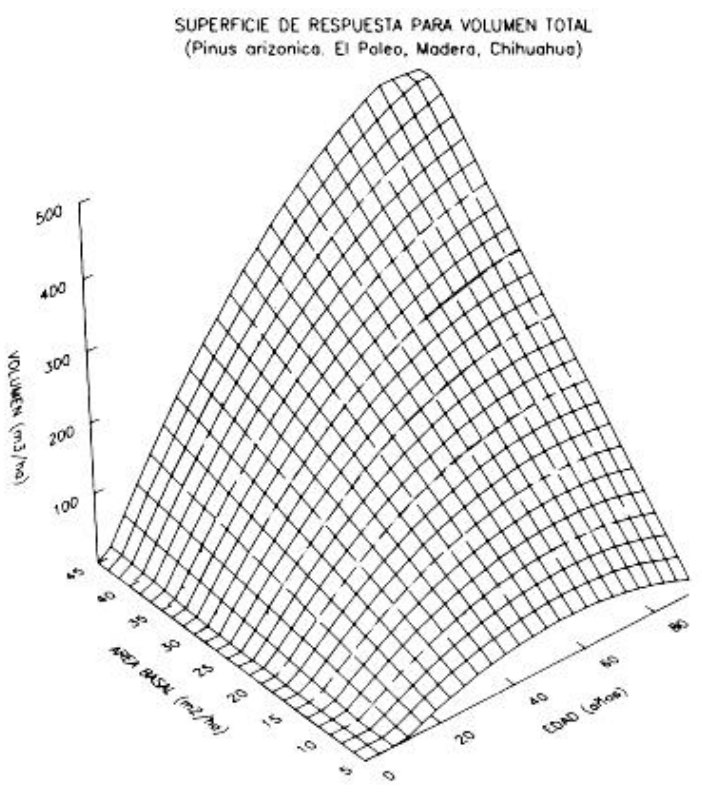

Figura 5. Relación entre el volumen total, el área basal y la edad. IS $=23 \mathrm{~m}$.

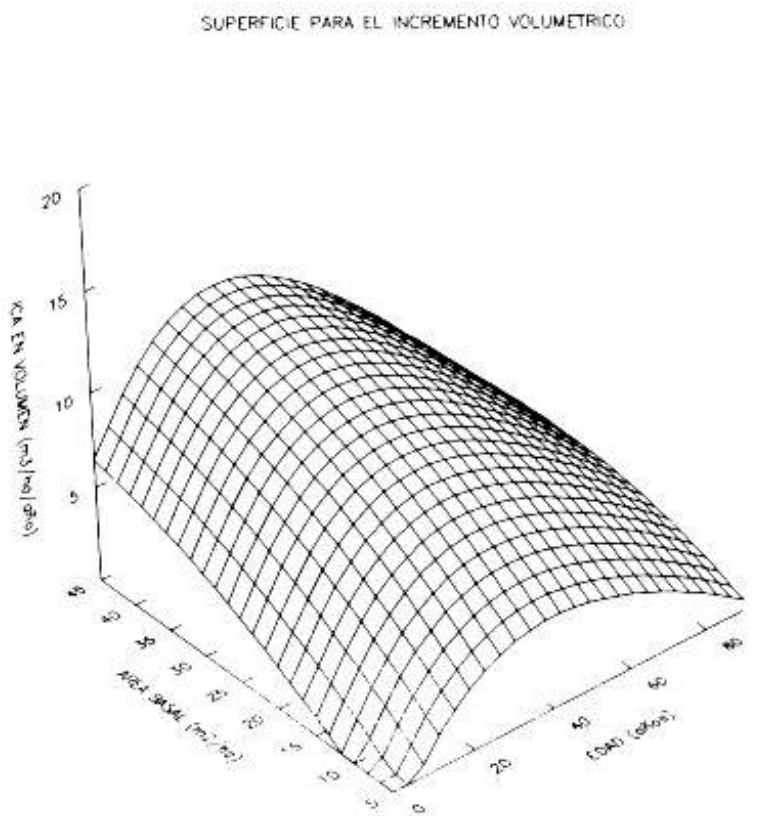

Figura 7. Relación entre el incremento anual en volumen, el área basal/hectárea y la edad. IS $=23 \mathrm{~m}$.
SUPERACIE OEL. INCREMENTO WOLUMETRCO

(Pinus arizonica. El Paleo, Modero, Chin)

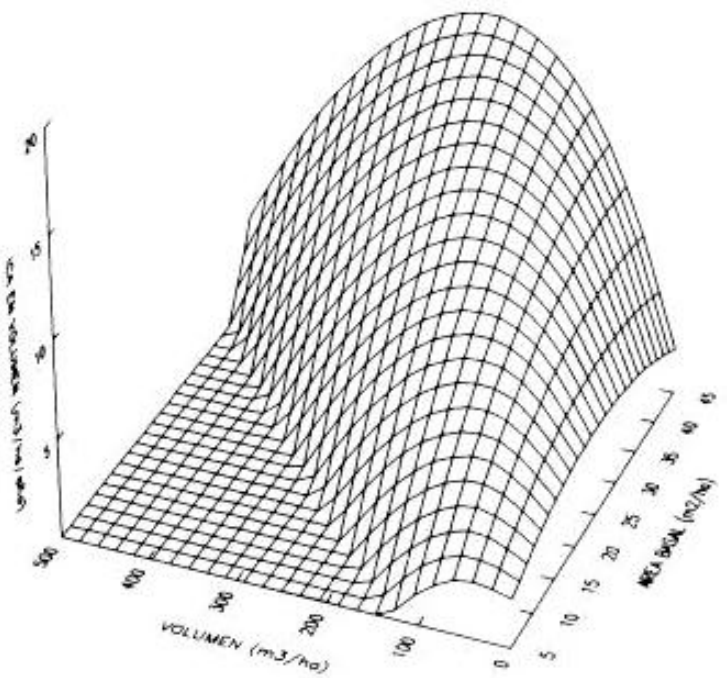

Figura 6. Relación entre el incremento anual en volumen, el volumen/hectárea y el área basal/hectárea. $\mathrm{IS}=23 \mathrm{~m}$.

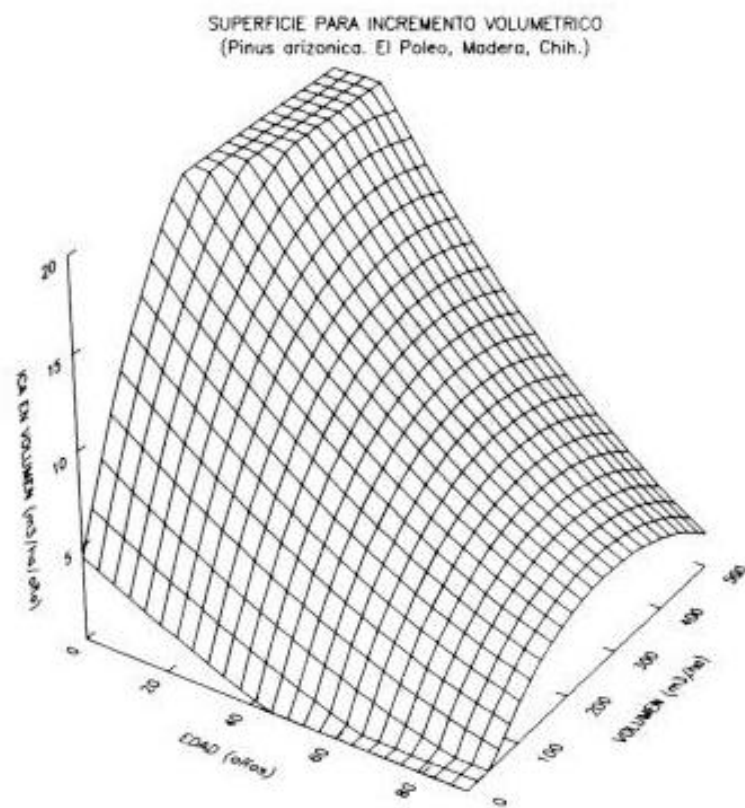

Figura 8. Relación entre el incremento anual en volumen, la edad y las existencias por hectárea. $\mathrm{IS}=23 \mathrm{~m}$. 


\section{CONCLUSIONES Y SUGERENCIAS}

Tomando en cuenta las limitaciones del presente análisis de rendimientos maderables, se arribó a las siguientes consideraciones:

El crecimiento y el rendimiento maderable son fenómenos interdependientes biológicamente, por lo que deben ser modelados matemáticamente atendiendo a esta situación, con la mayor precisión posible. Por ende, debe existir compatibilidad matemática entre el incremento y el rendimiento maderable, manifiesta en las ecuaciones preparadas para estimarlos, como ocurrió en este caso.

Los resultados aquí obtenidos pueden apoyar las decisiones que sobre intervenciones silvícolas se realicen en el área de El Poleo, ya que permiten evaluar, aún de manera inicial, algunas relaciones existentes entre la densidad, la productividad y la producción, válidas y aplicables únicamente a la especie y condición ambiental de interés (Fig. 2 y 3); mismas que no deben tomarse en forma dogmática, ni generalizarse para toda la UCoDeFo No. 2 de Chihuahua.

Estos resultados pueden ser usados también para modelar la respuesta del rendimiento maderable, producto de la interacción entre el control de la densidad y la capacidad productiva del sitio y, se piensa, auxiliar en la estimación de la producción, la evaluación inicial de tratamientos silvícolas alternativos, la valoración preliminar de rodales, el diseño de regímenes silvícolas y, entre otros, el control del rendimiento de madera; en todos los casos, únicamente para las condiciones de la mesa de El Poleo, Chih.

El rezago patente del manejo silvícola de coníferas en México, resultado del énfasis puesto en la toma de información semidetallada de "corte transversal", y no en el análisis de registros históricos, puede empezar a revertirse en la medida en que se cuente con la información que permita construir modelos dasométricos de pronósticos, indispensables para ello.

\section{RECONOCIMIENTOS}

Las gráficas $2 a$ y b y $3 a, b$ y c, fueron modificadas para su presentación por el Ing. Ignacio Salomón Quintana.

\section{REFERENCIAS}

Alder, D. 1979. A distance-independient tree model for exotic conifer plantations in East Africa. For. Sci.25(1): 59-71.

Clutter, J. L. 1963. Compatible growth and yield models for loblolly pine. For. Sci. 9(3):354-371.

Clutter, J. L., J.C. Forston, L.V. Pienaar, G.H. Brister y R.L. Bailey. 1983. Timber management: A quantitative approach. Wiley, New York. 333 p.

Salmon M., J.J. 1980. Influencia de la precipitación invernal en el crecimiento en altura de los pinos del grupo ponderosa en Chihuahua. Tesis. Universidad Autónoma Chapingo. 149 p.

Zepeda B., E.M., S. Veruette B., O. Estrada M. y S. Esparza P. 1990. Curvas polimórficas de índice de sitio de edad base invariante, para tres especies de pino del noroeste de Chihuahua. Universidad Autónoma Chapingo. División de Ciencias Forestales. Serie técnica. Bol. Téc. No. 25. 40 p.

Zepeda B., E.M., A. Domínguez P. y O. Estrada A M. 1994a. Ecuaciones para estimar rendimientos 
maderables de Pinus arizonica Engl., en el noroeste de Chihuahua. Resúmenes de Ponencias. II Reunión Nacional de Silvicultura y Manejo de Recursos Forestales. CP. Montecillo, Méx p: 44.
Zepeda B., E.M. y A. Domínguez P. 1994b. Rendimientos maderables de Pinus arizonica Engl., en El Poleo, Chihuahua. Resúmenes de Ponencias. II Reunión Nacional de Silvicultura y Manejo de Recursos Forestales. CP. Montecillo, Méx. p: 45. 